\title{
The impact of the national clinical outcome review programmes in England: a review of the evidence
}

\author{
Authors: Pauline Heslop, ${ }^{\mathrm{A}}$ Elena Baker-Glenn, ${ }^{\mathrm{B}}$ Peter Fleming, ${ }^{\mathrm{C}}$ Marian Knight, ${ }^{\mathrm{D}}$ Marisa Mason, ${ }^{\mathrm{E}}$ Pauline Turnbull ${ }^{\mathrm{F}}$ \\ and Clare Wade ${ }^{G}$
}

\section{Background}

There is a lack of evidence about the effectiveness of the national clinical outcome review programmes in England.

\section{Methods}

We undertook a scoping review of the published literature for evidence of the impact of any of the current programmes or their predecessors, and asked programme leads to share examples of the impact of their work. Data were thematically analysed.

\section{Findings}

Evidence about impact related to clinicians' awareness and practice, structural aspects of healthcare, processes of care and patient outcomes.

\section{Conclusions}

The national clinical outcome review programmes appear to have had significant impact, but none are funded to assess the outcome and impact of the recommendations they make or to deliver a programme of change. There is no structured and systematic way in which the findings and recommendations of each programme are taken forward, nor in which the findings from across programmes are collated and considered.

KEYWORDS: Clinical outcome review programmes, mortality review programmes, impact, effectiveness, service improvement

DOI: 10.7861/clinmed.2019-0359

\section{Background}

Mortality reviews afford the opportunity to examine the circumstances leading to the death of a person. Mortality and

Authors: ${ }^{\text {A }}$ professor of intellectual disabilities research, University of Bristol, Bristol, UK; ${ }^{B}$ national medical director's clinical fellow 2016/17, Royal College of Psychiatrists, London, UK; Cprofessor of infant health and developmental physiology, University of Bristol, Bristol, UK; D professor of maternal and child population health, University of Oxford, Oxford, UK; ${ }^{\text {E}}$ chief executive, National Confidential Enquiry into Patient Outcome and Death, London, UK; Froject director, National Confidential Inquiry into Suicide and Safety in Mental Health, Manchester, UK; ${ }^{G}$ head of patient safety, Royal College of Physicians, London, UK morbidity (MM) meetings have, for a long time, offered the opportunity for medical staff to review and learn from medical error, adverse events and in-hospital deaths with a view to changing practice as needed. Although common in medical services worldwide, the format and effectiveness of such meetings varies considerably. ${ }^{1,2} \mathrm{MM}$ meetings often lack the key characteristics needed to learn from medical and surgical incidents and improve patient safety: the ability to draw on the input of all staff involved in the incident; use of a structured framework to investigate the underlying contributing factors; and assign responsibility for management and follow up of recommendations. ${ }^{3} \mathrm{~A}$ recent systematic review concluded that peer-reviewed evidence of patient-centred outcomes as a result of the MM process is extremely limited. ${ }^{4}$

Concerns about the objectivity of those attending MM meetings and the heterogeneity of assessment between primary local review and external panel review have led to the development of more systematic, standardised mortality review processes by multidisciplinary teams. ${ }^{5-7}$ These are more likely than MM meetings to consider broader concerns such as interagency working, communication and systems-based problems and to be more accountable for taking corrective action should adverse events arise. ${ }^{8,9}$ The strength of national mortality reviews is the ability to review deaths, both individually and as aggregated data. Aggregated data allow identification of broader patterns, repeated errors, and trends that may not otherwise be identified at local level and, with appropriate policy changes and interventions, have the potential to lead to more effective improvements in outcomes. 10

\section{National clinical outcome review programmes in England}

The shift to national surveillance and response that informs the wider health and care system has been made in the UK through confidential enquiries, now known as the national clinical outcome review programmes. ${ }^{11}$

Broadly speaking, the key objectives of the clinical outcome review programmes are to improve the overall quality of care by identifying potentially avoidable factors that result in poor outcomes and making recommendations for prevention. Core components of successful programmes include strong government commitment and funding; a professional requirement to participate; adequate legal frameworks for the identification 
and notification of relevant cases; a 'no shame, no blame' culture; systematic processes in place for identifying remediable actions and publishing and disseminating reports; and a good working relationship with, but clear independence from, relevant government departments to ensure implementation of national recommendations. ${ }^{12}$

Over a decade ago, Sidebotham et al suggested there was a need for research into the outcomes of child death reviews, a suggestion echoed by Angelow and Black, who argued that although clinicians appear to have a strong belief in the value of clinical outcome review programmes, there was a lack of evidence of the impact of recommendations emanating from them. ${ }^{13,14}$ In this paper we provide evidence gathered from each of the national clinical outcome review programmes in England and a scoping review of the literature to identify and report on the impact of the current national clinical outcome review programmes in England.

\section{Methods}

In 2018, we approached the lead of each of the national clinical outcome review programmes in England to share examples of the impact of their work. In addition, we undertook a scoping review of the published literature for evidence of the impact of any of the current programmes or their predecessors, including confidential enquiries. Databases searched included Medline, CINAHL and PsychINFO. The search was conducted in 2018 and drew on evidence of impact between 2000 and 2018. Data was thematically analysed, the focus being the uptake of recommendations and the impact of this on the care received by specific populations.

\section{Findings}

Table 1 outlines the national clinical outcome review programmes included in the study. All are currently operating in England, apart from the National Mortality Case Record Review programme which finished in June 2019.

Evidence of the impact of the programmes is presented below in relation to clinicians' awareness and practice; structural aspects of healthcare; processes of care; and patient outcomes.

\section{Clinicians' awareness and practice}

The impact of the national clinical outcome review programmes on clinicians' awareness and practice was highlighted by

\section{Table 1. The national clinical outcome review programmes in England}

Programme name

Maternal, Newborn and Infant Clinical Outcome Review Programme

Medical and Surgical

Clinical Outcome Review Programme

Mental Health Clinical

Outcome Review

Programme

Child Health Clinical

Outcome Review

Programme

Confidential Inquiry into premature deaths of people with learning disabilities

Learning Disability

Mortality Review

Programme

National Mortality

Case Record Review

Programme

National Child Mortality

\section{Established and any developments}

1952: Maternal deaths

1993: Stillbirths and deaths in infancy

2003: Maternal and child health

2010: Mothers and babies

1988

2010-2013

2016-2019

Database

\section{Description of current} programme

Conducts surveillance and investigates the causes of materna deaths, stillbirths and infant deaths

Highlights remediable factors in the care of patients across medical and surgical clinical topic areas

Examines suicide by people who had been in contact with secondary and specialist mental health services in the previous 12 months

Uses case-note review to examine specific topics relating to child health

Reviewed the deaths of 247 people with learning disabilities, and 58 comparator cases in five (former) primary care trusts.

Supports local areas to review the deaths of all people with learning disabilities.

Introduced a standardised methodology for reviewing case records of adult patients who have died in acute general hospitals in England and Scotland

Collects core information about all children in England who die before their 18th birthday

\section{Lead agency}

Mothers and Babies:

Reducing Risk through Audits and Confidential Enquiries across the UK, Universities of Oxford and Leicester

National Confidential Enquiry into Patient Outcome and Death, London National Confidential Inquiry into Suicide and Safety in Mental Health, University of Manchester

National Confidential Enquiry into Patient Outcome and Death, London University of Bristol

University of Bristol

Royal College of Physicians

University of Bristol (with University of Oxford and University College London). 
Case example 1. Clinical care compromised by lack of awareness of the specific needs of people with learning disabilities ${ }^{15}$

When a woman was admitted to hospital, her medical notes on admission described her as 'mute, aphasic and having learning difficulties'. A plan was made to stop any treatment and to transfer her back to the nursing home for end-of-life care. The nursing home manager and her general practitioner disagreed with the decision not to treat her, believing that this was on the basis that the hospital team lacked awareness of her needs and any reasonable adjustments that could be put in place for her. They insisted on the Mental Capacity Act being followed, and a best interest meeting was held that afternoon, attended by the hospital doctors involved, the nursing home manager, the hospital learning disability liaison nurse and four members of the person's family. Active treatment was resumed as a result of the decisions made, and the person had improved greatly within 48 hours. She was discharged 2-3 weeks later and lived for more than another year before dying peacefully at home.

the Confidential Inquiry into deaths of people with learning disabilities (CIPOLD) from 2010-2012. ${ }^{15}$ A 'ripple effect' of the impact of being involved with CIPOLD was reported, with changes in professionals' knowledge, awareness and practice in relation to supporting people with learning disabilities; increased confidence in challenging what was felt to be inappropriate care; and supporting other practitioners to take a more personcentred, holistic approach. The review process was felt to have raised clinician's awareness about specialist learning disability services and sources of advice; to have reminded clinicians about relevant legislation in relation to the care of people with learning disabilities; and to have refreshed clinician's knowledge about risk management and safeguarding procedures. ${ }^{15}$

One of CIPOLD's recommendations was for the ongoing surveillance and review of deaths of people with learning disabilities and the English Learning Disabilities Mortality Review (LeDeR) programme was launched in 2015. ${ }^{16}$ Both CIPOLD and the LeDeR programme have highlighted specific examples of clinical care that was compromised by lack of awareness of the specific needs of people with learning disabilities (Case example 1). ${ }^{15}$ Hence, one of the early recommendations of the LeDeR programme was for the introduction of mandatory training for health and social care staff supporting people with learning disabilities to raise their awareness and improve practice. The government has since consulted widely on options for delivering this. ${ }^{17}$

The most recently established clinical outcome review programme is the Child Death Mortality Database. One of the immediate effects of its implementation has been the identification of gaps in the national process for reviewing deaths of children and recognition of the limited (or sometimes very poor) communication with bereaved families in some areas. Work has already begun to help develop and implement ways of improving these aspects of care.

\section{Structural aspects of healthcare}

Structural aspects of healthcare include those such as staffing levels, the availability and organisation of facilities, and the
Case example 2. Clinical example of the quality of hospital care provided to a trauma victim ${ }^{18}$

A middle-aged patient was admitted to the minor injuries section of an emergency department at 22:15 hours following a fall onto the back of their head. Admission Glasgow Coma Score (GCS) was documented as 6. Despite the low GCS and the history of head trauma there was no ambulance pre-alert and no trauma team response. The patient was placed in a cubical, commenced on neurological observations, given $15 \mathrm{~L} / \mathrm{min}$ of oxygen and placed in the recovery position. No medical review happened until 23:40 when, after a tonic-clonic seizure, the patient was seen by a senior house officer (SHO). The patient's GCS was recorded as 3 after the seizure. No investigation or intervention occurred at this time. The patient had a subsequent seizure at 00:05 and was given lorazepam at that time. Finally, at 01:00 the patient was taken for computed tomography (CT). The GCS was still recorded as 3. The patient was not intubated and was escorted to radiology by the surgical SHO. The CT revealed a large intracerebral haemorrhage with significant midline shift. The patient was transferred back to the emergency department and at 01:30 the patient was referred to the anaesthetic $\mathrm{SHO}$. The anaesthetic $\mathrm{SHO}$ contacted the specialist registrar on call for anaesthesia and following their attendance the patient was intubated at 03:00. The patient subsequently died from severe brain injury.

The reviewers commented on the lack of any formal trauma response, delays to medical review and lack of a consultant-led service leading to a series of delays and poor-quality care which impacted on the outcome for this patient.

local introduction of guidelines. One example of the impact of a national clinical outcome review programme on structural aspects of healthcare is provided by the National Confidential Enquiry into Patient Outcome and Death review of trauma care. ${ }^{18}$ The study was established because of concerns that the quality of hospital care provided to trauma victims was not of a consistently high standard across the UK and that some hospitals had insufficient experience in dealing with severe trauma (Case example 2). ${ }^{18}$

The Trauma: Who cares? study recommended significant changes to the structural delivery of trauma care with the need for designated major trauma centres and a verification process to quality assure the delivery of trauma care. ${ }^{18}$ Following the publication of the report, a national clinical director was appointed to embed the recommendations and the National Audit Office recommended the development of regional trauma networks in England. The first major trauma centre opened in England in 2012 and there are now 27 in England. All operate within local trauma networks, ensuring that patients are treated at the most appropriate place for their injuries, but then return closer to home for ongoing care when appropriate. A recent report suggests that since the creation of major trauma centres, the lives of 1,600 patients with severe injuries have been saved. ${ }^{19}$

The Mothers and Babies: Reducing Risk through Audits and Confidential Enquiries across the UK (MBRRACE-UK) national programme of maternal mortality and morbidity surveillance and confidential enquiries has led to major structural changes in care for pregnant women at higher risk. The work has shown that two-thirds of the population attributable risk of maternal 
Case example 3. Clinical example of symptoms of medical disorder being attributed to pregnancy ${ }^{20}$

An older woman had an uneventful pregnancy until 34 weeks when she developed chest pain. The pain had been off and on over 3 days and radiated into her back and left arm. The woman smoked, had a family history of ischaemic heart disease and a history of hypertension. She had been given entonox in the ambulance which is known to treat ischaemic pain. When she was assessed in the emergency department, no one asked about her risk factors for coronary disease. Despite abnormal electrocardiography, she had no further investigations. The obstetric team was not contacted. She was discharged home from the emergency department and found dead in bed the following day. Extensive coronary artery atherosclerosis and a thrombosed left anterior descending artery were found at postmortem examination.

death is associated with medical comorbidities (Case example 3), frequently when concerning symptoms of medical disorders are attributed to pregnancy. ${ }^{20}$

Another key finding of the MBRRACE-UK national programme of maternal mortality and morbidity surveillance highlighted maternal suicide as an important cause of death in the year postpartum, emphasising the risks of deprescribing medication in pregnancy (Case example 4). ${ }^{20}$

These findings led to the introduction of new maternal medicine networks in England, announced in November 2017, the expansion of access to specialist perinatal mental health services proposed in NHS England's The NHS Long Term Plan, and new funding of $£ 50$ million for perinatal mental health services in Scotland. ${ }^{21-23}$

The new maternal medicine networks will ensure that there is a trained obstetric physician in each of 12 regions of England, with an associated network to ensure expert care for pregnant women with medical comorbidities. The additional perinatal mental health funding will ensure all pregnant and postpartum women are able

Case example 4. Clinical example of maternal suicide $^{20}$

A woman took her life by violent means in her third trimester. She had a previous history of anxiety and depression, with depression in a previous postnatal period. She had been prescribed venlafaxine prior to the pregnancy to good effect, but it was stopped on discovering the pregnancy. No alternative was suggested and there does not appear to have been any specialist service within her area

She developed worsening anxiety, and then depression, with a range of physical complaints, poor coping and suicidal ideation. As her symptoms worsened, she was referred to a low intensity psychological therapies service. She returned to her general practitioner (GP) asking to restart her previously effective venlafaxine. It is clear from the consultation notes that her GP was very reluctant to prescribe and placed responsibility for the decision entirely on the woman, documenting an explanation of the risks, but not the benefits, of taking medication. She died a week later on the day she was due to undergo a mental health assessment.
Case example 5. Clinical example of failing in the management of acute kidney injury ${ }^{24}$

An elderly patient was admitted to hospital with a history of lethargy and confusion for a few days. It was noted on admission that the patient had acute kidney injury. A urinary catheter was inserted; however, after initially draining $200 \mathrm{~mL}$ there was no further urine output. There was no clear working diagnosis and a plan to modify existing drug therapy (to stop potential nephrotoxins) and volume resuscitate the patient was made. No further investigations were ordered. Twenty-four hours later urea and creatinine had worsened despite the above treatment. Following consultant review a renal ultrasound was ordered. This revealed bilateral hydronephroses. Prior to decompression of the renal tract the patient suffered a cardiac arrest and died.

The reviewers commented that the delay in imaging the renal tract may have contributed to death and that earlier intervention may have allowed recovery of renal function.

to access specialist perinatal mental health services when required. Prevention of maternal deaths in association with medical and mental health comorbidities has the capacity to ensure achievement of the Department of Health and Social Care's ambition in England to reduce maternal deaths by $50 \%$ by 2025 .

\section{The delivery of care}

A further way in which the impact of the national clinical outcome review programme is recognised is through the process or delivery of care. One exemplar of this is the National Confidential Enquiry into Patient Outcome and Death (NCEPOD) review of acute kidney injury published in 2009. ${ }^{24}$ The NCEPOD study identified systematic failings in the recognition and management of acute kidney injury by clinicians (Case example 5), and a failure to recognise the complications of the condition. ${ }^{24}$

The 'landmark' enquiry stimulated a range of initiatives highlighting acute kidney injury as a national priority area for action. ${ }^{25}$ It informed a referral from the Department of Health and Social Care to the National Institute for Health and Care Excellence (NICE) to develop its first guideline on acute kidney injury, subsequently published in $2013 .{ }^{26}$ In 2016, the NHS 'Think Kidneys' campaign programme for tackling acute kidney injury was introduced, with the aim of improving care for patients. ${ }^{27}$ In 2017 the Royal College of General Practitioners introduced the Acute Kidney Injury Toolkit as part of a quality improvement initiative. ${ }^{28}$ Part of the toolkit includes an interactive case-based e-learning module covering the recognition, assessment, and management of acute kidney injury in the community, with practical advice for clinicians about when to refer and how to follow-up patients after an episode of acute kidney injury.

Evidence of the effectiveness of improved delivery of care to people with acute kidney injury has subsequently been reported by a number of acute hospitals. ${ }^{25,29}$

\section{Patient outcomes}

The longest running of the confidential enquiries is that of the UK Confidential Enquiry into Maternal Deaths, which was started more than 60 years ago. During this time, maternal mortality rates have fallen 10 -fold. ${ }^{30} \mathrm{~A}$ large proportion of this reduction has been due to 
Case example 6. Clinical example of missed opportunities for intervention ${ }^{30}$

A woman with risk factors for pre-eclampsia presented at term with an abnormal fetal heart rate trace. She had an emergency caesarean birth. Postnatally she had significantly abnormal renal, liver and clotting function but there was no direct consultant obstetric involvement and the severity of her illness was not recognised until very late. There were differing opinions about transfer to intensive care and despite multiorgan problems she remained in the obstetric high dependency unit. She continued to deteriorate with markedly abnormal liver function, but at no point was a gastroenterology or hepatology opinion sought. She eventually died from her liver failure several days after giving birth. There was no post-mortem or serious incident review carried out. At all stages the severity of her underlying pathology was underestimated and opportunities to escalate her care were missed.

the implementation of recommendations from reviews of maternal deaths that have highlighted the importance of routine screening, blood pressure control and fluid management in pregnancy (Case example 6). ${ }^{30}$ The impact of this has been a reduction in deaths from hypertensive disorders of pregnancy to fewer than one in every million women giving birth in the UK. ${ }^{20}$ Overall, the past 60 years have seen a dramatic reduction in the maternal mortality rate, such that maternal deaths are now very rare. ${ }^{30}$

The National Mortality Case Record Review programme (2016-2019) retrospectively reviewed the quality of a deceased patient's care from hospital admission to death using a validated Structured Judgment Review (SJR) tool for case-note review. Although relatively new, clinicians have reported that having access to a validated, standardised methodology has given them the confidence to review the care delivered to adult acute patients in a robust way and use this as the basis for locally led quality improvement initiatives. ${ }^{31}$ As an example, reviews at one NHS foundation trust were estimated to have resulted in a reduction in the standardised mortality rate for deaths from acute cerebrovascular disease from 130 in 2016 to 114 the following year (Case example 7). The SJR tool has been adapted for use

Case example 7. Using a structured review tool to lead to improved patient outcomes

In the trust concerned, there was a high standardised mortality rate for deaths from acute cerebrovascular disease. The trust's aim was to identify lessons and implement learning within 8 weeks. The actions taken by the trust were:

to identify index cases and compare with a similar time period one year later

to conduct demographic analysis

to select 30 cases for Structured Judgement Review including all deaths in patients less than 60 years old

to share findings with the clinical commissioning group and general practices.

The trust's standardised mortality rate for deaths from acute cerebrovascular disease was 130 initially but reduced to 114 a year later.
Case example 8. Report of inquest into death of a

person in a hospital bathroom ${ }^{35}$

A woman was found hanging in a hospital bathroom 8 months after parts of the room were identified as potential ligature points, an inquest heard. The 52-year-old, who had bipolar disorder, had been sectioned under the Mental Health Act. The jury inquest concluded she had died as a result of an accident contributed to by neglect.

Eight months prior, staff had carried out an audit of potential ligature points in the hospital and identified parts of the lockable bathroom on the mental health ward as being potential ligature points. An application for funding was made to carry out remedial works, but no action had been taken by the time of the person's death.

within mental health trusts and the ambulance service and it has successfully identified areas of good practice in addition to areas for improvement in patient care. ${ }^{32,33}$ Participation in such panel discussions has been recognised by the royal colleges as an important part of continuing professional development for clinical professionals.

Patient outcomes have also improved as a result of the National Confidential Inquiry into Suicide and Safety in Mental Health (NCISH) which has collected in-depth information on suicides in the UK since 1996. The recommendation to remove all noncollapsible curtain rails and its adoption as an NHS 'never event' has been estimated to have resulted in a $60 \%$ fall in deaths by hanging in inpatient wards. NCISH demonstrated that mental health service implementation of their recommendations was associated with lower suicide rates than in non-implementing services (Case example 8). ${ }^{34,35}$ In 2018, Public Health England and NHS England announced a $£ 25$ million 3-year programme of local suicide prevention quality improvement work based on NCISH recommendations.

\section{Discussion and conclusions}

The national clinical outcome review programmes have evolved over time and appear to have had significant impact since their inception, including saving lives and resources, and generally improving the quality of care provided to patients. The in-depth nature of their work can add weight to and complement other audits or national data collections by getting to the detail of what happened and what should have happened in a more informed way, resulting in more targeted recommendations for improvement. For the work of reviewing deaths to be accepted as valid, the core pillars of independence, confidentiality and trust appear to be crucial.

What is apparent from this review of the impact of the clinical outcome review programmes is that, although individual reflection can result in local change quite quickly, it takes much longer to embed national recommendations and demonstrate changeseveral years in some cases. Thus, although some of the longeststanding clinical outcome review programmes can demonstrate considerable impact at national level, this is less likely to be the case for some of the newer programmes.

Each of the clinical outcome review programmes is contracted to deliver a process of information gathering only; none are 
currently funded to assess the outcome and impact of the recommendations they make or to deliver a programme of change. What happens to the recommendations made by each of the programmes is therefore dependent on political will or committed practitioners. There is no structured and systematic way in which the findings and recommendations of each programme are taken forward, nor in which the findings from across programmes are collated and considered. This appears to be a significant gap in their effectiveness.

In our view, there are several ways in which the clinical outcome review programmes could be strengthened. First is the need for a national mortality oversight body to streamline mortality review processes where needed, draw together the findings and recommendations across the mortality review programmes, prioritise recommendations, and oversee their implementation. Second is the need to draw on the expertise of implementation science to consider the complex systems into which recommendations are made and improve the effectiveness of the recommendations taken forward into service improvements. Third we need to carefully balance the desire to collect more information with the resources needed to effect change and measure the impact of this. Reviews of deaths in themselves may be helpful but should not be at the expense of actions taken to prevent other early deaths. There is a moral and ethical dimension to this. If programmes are funded purely to gather and share information, they need to be assured that identified deficiencies in care are going to be addressed.

\section{Acknowledgements}

We would like to acknowledge the role of the Healthcare Quality Improvement Partnership (HQIP). HQIP is led by a consortium of the Academy of Medical Royal Colleges, the Royal College of Nursing and National Voices, and holds the contract to commission, manage and develop the national clinical outcome review programmes. HQIP is funded by NHS England, the Welsh Government and in some cases other devolved administrations. For more information see www.hqip.org. uk/national-programmes.

\section{References}

1 Xiong $X$, Johnson $T$, Jayaraman $D$ et al. At the crossroad with morbidity and mortality conferences: Lessons learned through a narrative systematic review. Can J Gastroenterol Hepatol 2016;2016:7679196.

2 Lecoanet A, Vidal-Trecan G, Prate F et al. Assessment of the contribution of morbidity and mortality conferences to quality and safety improvement: a survey of participants' perceptions. BMC Health Serv Res 2016;16:176.

3 Berenholtz SM, Hartsell TL, Pronovost PJ. Learning from defects to enhance morbidity and mortality conferences. Am J Med Qual 2009;24:3.

4 Joseph CW, Garrubba ML, Melder AM. Informing best practice for conducting morbidity and mortality reviews: a literature review. Australian Health Review 2018;42:248-57.

5 Gupta M, Fuchs B, Cutilli C et al. Preventable mortality: does the perspective matter when determining preventability? J Surg Res 2013;184:54-60.

6 Bender LC, Klingensmith ME, Freeman BD et al. Anonymous group peer review in surgery morbidity and mortality conference. Am J Surg 2009;198:270-6.

7 Masson VL, Farquhar CM, Sadler LC. Validation of local review for the identification of contributory factors and potentially avoidable perinatal deaths. Aust N Z J Obstet Gynaecol 2016;56:282-8.
8 Higginson J, Walters R, Fulop N. Mortality and morbidity meetings: an untapped resource for improving the governance of patient safety? BMJ Qual Saf 2012;21:576e585.

9 Travaglia J, Debono D. Mortality and morbidity reviews: a comprehensive review of the literature. Sydney: University of New South Wales, 2009.

10 Clark SL, Belfort MA. The case for a national maternal mortality review committee. Obstet Gynecol 2017;130:198-202.

11 Healthcare Quality Improvement Partnership. A-Z of Clinical Outcome Review Programmes. HQIP. www.hqip.org.uk/clinicaloutcome-review-programmes/\#.Xt4PJTpKhPZ

12 Smith H, Ameh C, Roos N, Mathai M, van den Broek N. Implementing maternal death surveillance and response: a review of lessons from country case studies. BMC Pregnancy Childbirth 2017;17:233.

13 Sidebotham P, Fox J, Horwath J, Powell C, Perwez S. Preventing childhood deaths: a study of 'early starter' child death overview panels in England. London: Department for Children, Schools and Families, 2008.

14 Angelow A, Black N. The use and impact of national confidential enquiries in high-income countries. BMJ Qual Saf 2010;20: $38 \mathrm{e} 45$.

15 Heslop P, Blair P, Fleming P et al. The Confidential Inquiry into premature deaths of people with learning disabilities (CIPOLD). Bristol: University of Bristol, 2013. www.bristol.ac.uk/cipold/reports [Accessed 02 September 2019].

16 University of Bristol. Learning Disabilities Mortality Review (LeDeR) programme Annual Report. Bristol: University of Bristol, 2017. www.bristol.ac.uk/media-library/sites/sps/leder/leder_annual_ report_2016-2017.pdf [Accessed 02 September 2019].

17 Department of Health and Social Care. Learning disability and autism training for health and care staff. A consultation. DHSC, 2019. www.gov.uk/government/consultations/learning-disabilityand-autism-training-for-health-and-care-staff [Accessed 02 September 2019].

18 National Confidential Enquiry into Patient Outcome and Death. Trauma: Who cares? NCEPOD, 2007. www.ncepod. org.uk/2007report2/Downloads/SIP_report.pdf [Accessed 02 September 2019].

19 Moran G, Lecky F, Bouamra O et al. Changing the system-major trauma patients and their outcomes in the NHS (England) 200817. EClinicalMedicine 2018;2-3:13-21.

20 Knight M, Nair M, Tuffnell D et al. Saving Lives, Improving Mothers' Care-Surveillance of Maternal Deaths in the UK 2012-14 and Lessons Learned to Inform Maternity Care from the UK and Ireland Confidential Enquiries into Maternal Deaths and Morbidity 2009-14. Oxford: University of Oxford, 2016.

21 Department of Health. Safer Maternity Care. The national maternity safety strategy - Progress and next steps. DoH, 2017. https:// assets.publishing.service.gov.uk/government/uploads/system/ uploads/attachment_data/file/662969/Safer_maternity_care_-progress_and_next_steps.pdf [Accessed 02 September 2019].

22 NHS England. The NHS Long Term Plan. NHS, 2019. https://www. longtermplan.nhs.uk/wp-content/uploads/2019/01/nhs-long-termplan-june-2019.pdf [Accessed 02 September 2019].

23 Scottish Government, NHS Scotland. Delivering effective services: Needs assessment and service recommendations for specialist and universal perinatal mental health services. NHS Scotland, 2019. https://maternalmentalhealthscotland.org.uk/wp-content/ uploads/2019/03/Delivering-Effective-Services.pdf [Accessed 02 September 2019].

24 National Confidential Enquiry into Patient Outcome and Death. Acute Kidney Injury: Adding Insult to Injury. London: NCEPOD, 2009. www.ncepod.org.uk/2009report1/Downloads/AKI_report.pdf [Accessed 02 September 2019].

25 Sykes L, Sinha S, Hegarty ] et al. Reducing acute kidney injury incidence and progression in a large teaching hospital. BMJ Open Qual 2018;7:e000308. 
26 National Institute for Health and Care Excellence. Acute kidney injury: prevention, detection and management: Clinical guideline [CG169]. NICE, 2013. www.nice.org.uk/guidance/cg169

27 NHS. Think Kidneys. NHS. www.thinkkidneys.nhs.uk

28 Royal College of General Practitioners. Acute Kidney Injury Toolkit. RCGP. www.rcgp.org.uk/clinical-and-research/resources/toolkits/ acute-kidney-injury-toolkit.aspx

29 Chandrasekar T, Sharma A, Tennent L et al. A whole system approach to improving mortality associated with acute kidney injury. QJM 2017;110:657-66.

30 Knight M, Tuffnell D. Confidential Enquiry into Maternal Deaths and Morbidity. Clinical Obstet Gynecol 2018;61,2:347-58.

31 Gibson A, Wade C, Appalsawmy U, McCooe M, Atkinson A. National Mortality Case Record Review (NMCRR): Annual report 2018. London: Royal College of Physicians, 2018.

32 Royal College of Psychiatrists. Mortality Review Tool: Mortality Review Tool Guidance. RCPysch, 2019. www.rcpsych.ac.uk/ improving-care/campaigning-for-better-mental-health-policy/carereview-tool-for-mental-health-trusts [Accessed 02 September 2019].
33 National Quality Board. National guidance for ambulance trusts on Learning from Deaths. A framework for NHS ambulance trusts in England on identifying, reporting, reviewing and learning from deaths in care. NHS, 2019. www.england.nhs.uk/wp-content/ uploads/2019/07/learning-from-deaths-guidance-for-ambulancetrusts.pdf [Accessed 02 September 2019].

34 While D, Bickley H, Roscoe A et al. Implementation of mental health service recommendations in England and Wales and suicide rates, 1997-2006: a cross-sectional and before-and-after observational study. Lancet 2012;379:1005-12.

35 BBC News. Royal Glamorgan Hospital hanging death after risks found. BBC, 27 July 2018. www.bbc.co.uk/news/uk-wales-southeast-wales-44979101 [Accessed 17 October 2019].

Address for correspondence: Prof Pauline Heslop, University of Bristol School for Policy Studies, 8 Priory Road, Bristol BS8 1TZ, UK.

Email: pauline.heslop@bristol.ac.uk 